\title{
In-situ Process Control for Semiconductor Manufacturing
}

\author{
James H. Taylor \\ Department of Electrical \& Computer Engineering \\ University of New Brunswick \\ Fredericton, N. B. CANADA E3B 5A3 \\ email: jtaylor@unb.ca \\ Thomas K. Whidden \\ FiberOptic Gateways Inc. \\ Fredericton, N. B. CANADA \\ email: twhidden@nbnet.nb.ca \\ Zhao Xiaozhong \\ Dalhousie University Department of Engineering \\ Halifax, Nova Scotia, CANADA \\ email: xiaozhong_zhao@yahoo.ca
}

\begin{abstract}
There is a critical need for exact, real-time reaction control of the chemical vapor deposition (CVD) systems that are used for semiconductor device manufacture [1]. At present, the development of real-time control for reactions within production-style reactor configurations is hampered by a number of issues: The nature, concentrations and physical distributions of the chemical species within the deposition chamber must be measurable for effective feedback control. These parameters are virtually uncharacterized at this time, even for processes that have been in use for prolonged periods [2]. The chemical kinetic relationships underlying the fabrication processes, while they have been modeled in certain cases have, in most instances, not been experimentally confirmed. These models are especially needed to effectively control particle nucleation within CVD reactors. The lack of chemical data on these systems is, at least in part, due to the fact that reliable, suitably configured sensors have not been generally available.

The above impediments to real-time CVD reaction control are being eliminated. Fourier-transform infra-red (FT-IR) spectrometry is being used to provide the required sensing, and the chemical kinetic relationships involved in device manufacture are being understood and modeled. A prototype control system has been developed using an FTIR sensor to control the reaction chemistry for a specific CVD process, and a plan for extending and commercializing this technology has been created. These recent accomplishments are described in this paper.
\end{abstract}

\section{Introduction}

Chemical state sensing and sensor-based real-time control is in its infancy for the chemical vapor deposition (CVD) process tools that are used in microelectronic device fabrication. Chemical state sensing in today's generation of equipment is at best opportunistic and generally the available techniques are employed solely for trouble-shooting purposes (e.g., analysis of exhaust gases). Typically, control protocols in the current generation of CVD tools can be characterized as the open-loop execution of pre-specified and fixed recipes. While low-level feedback loops control the basic process variables such as temperature, pressure and gas flows, there is no attempt to monitor the chemical process in real time or to control the chemistry as it occurs within the reaction chamber. However, there is presently a general acknowledgement within the semiconductor industry that in situ and real-time chemical state sensing and control will be a requirement for the successful fabrication schemes for nanoscale device fabrication [1]. Consequently, there is now a significant effort ongoing for the development of such sensors and control strategies.

Two device requirements are forcing the transition to in situ sensing and real-time process control protocols in fabrication processes. One is the increasing restriction on selected material property specifications that are permissible in the different component thin films that are used to build ultra large scale integrated (ULSI) devices. As device geometries shrink, the allowable window on process variations for producing materials with acceptable electrical properties gets ever smaller. This requires that the CVD and etch processes that are used to form and pattern the thin film adopt ever more restrictive process control limits. Processes with the required degree of control for device fabrication in the unfolding ULSI era can only be achieved by using in situ sensing to provide data for real-time feedback control.

The second factor dictating this transition is that, in the near future many of the thin film processes used in device fabrications will undergo an unprecedented increase in chemical complexity. This is due to upcoming changes in the basic materials for the construction of advanced devices. CVD processes in the future will no longer use simple hydrides or halides in low-pressure reaction regimes. Most will em- 
ploy organometallic chemical systems and many will be forced to migrate to higher-pressure or plasma-enhanced reaction protocols to achieve production-worthy deposition rates. Such reaction regimes (and even many low-pressure regimes) have rich gas-phase chemistries that profoundly influence both the process and the film's material properties, thus requiring tight control during the device fabrication process. Figure 1 shows the simple precursor compounds of today $v s$ some organometallics that will be used in future CVD processes. It is believed that acceptable yields in device fabrication can only be achieved with an improved understanding of CVD chemistries and with new approaches to monitoring and controlling the deposition and etching reactions when using such complex chemical species.

Practical real-time control under the anticipated more restrictive process limits and with more chemically complex systems will require an improved understanding of the nature of both the gas and surface chemistries extant within CVD reactions. At present, the interrelationships between gas and surface chemical reaction mechanisms, thin film material properties, reactor physical parameters and the overall process conditions are only beginning to be understood. By way of example, one can consider the pyrolysis of silane to produce polycrystalline silicon: The process has been empirically studied and optimized many times in different applications and numerous papers on the chemical kinetics and reactor modeling have been published over the past 75 years (reference [2] is representative). Still, the relative importance of heterogeneous $v s$ homogeneous reactions to the CVD process continued to be debated in the literature as recently as $1997[3,4]$.

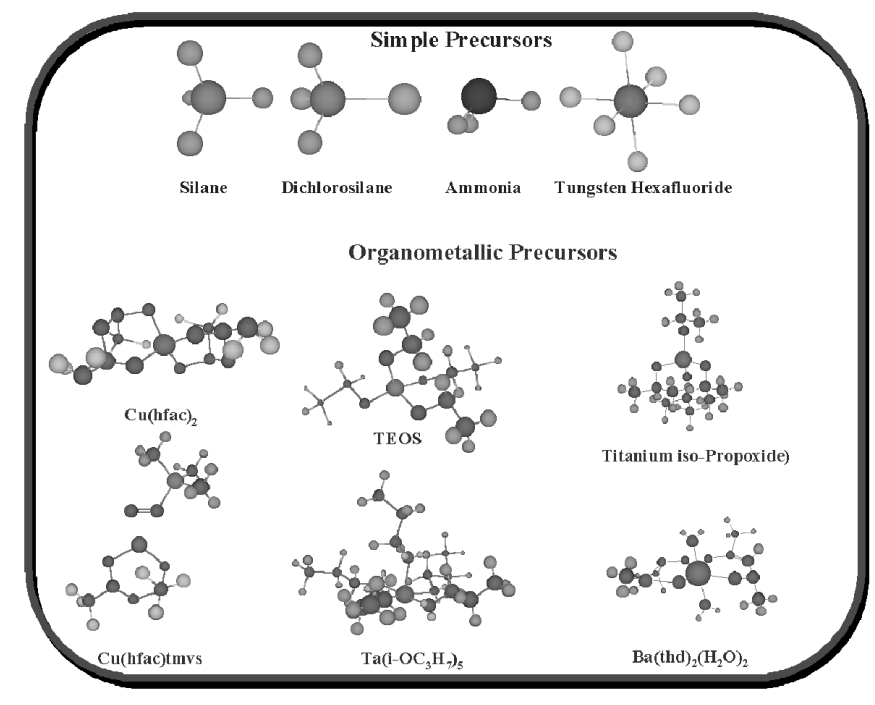

Figure 1: Current vs future precursor compounds

The introduction of in situ sensors into CVD systems can be the first step in the road to more advanced process control in these systems. The data from these sensors can be used in conventional feedback control systems that function in real-time. The initial development of these control systems can be simple. They can use well-established control protocols that employ regression techniques to identify the dynamic relations between manipulated variables (i.e. gas flows) and key process variables (chemical constituent concentrations). Algorithms can be designed and implemented that use a classical control strategy that is based on the identified regression model. Once these simple platforms have been developed, they can then serve as the basis for the use of more advanced control techniques such as intelligent/learning process control. This paper focusses on some of the issues that are related to sensing and control of CVD processes in an era of heightened materials property restrictions and increased chemical process complexity.

\section{In Situ Chemical State Sensing}

The previous discussion details some of the reasons for the new and exacting control requirements in deposition processing for silicon semiconductor device manufacture. Serious obstacles exist that will impact the industry's ability to achieve this degree of control. One of the most critical and immediate issues is the industry's need for effective in situ monitors of the chemical state in the reaction boundary layer above the wafer surface. A number of laboratory technologies for such monitoring exist; however, practical analytical tools (sensors) that are of use on the factory floor are not yet available.

The most sophisticated sensing tool that one might find in a production setting is usually a quadrupole mass spectrometer (residual gas analyzer or RGA). Typically, these are used for exhaust gas analysis when system upsets occur. Unfortunately, RGA detectors are physically ill suited to deposition environments, in that accumulated deposits on the filaments cause drift in the sensor response. This creates difficulties for process control schemes that are based on data from this type of sensor. In addition, RGAs cannot sample directly above a substrate without deleterious effects on the thin film and process.

Theoretically, in situ analyses using optical emission spectroscopy (OES) can provide information on the chemical state within chemical reactors using plasma excitation and, indeed, such sensors are beginning to be commonly employed for end-point detection in plasma etching processes. Unfortunately, it has been found in practice that chemically useful interpretations of the data from OES sensors that are placed on plasma enhanced CVD tools are notoriously difficult to obtain. More importantly in our context, thermal CVD systems cannot be analyzed using OES due to the general lack of optical emissions [7].

Finally, advanced techniques such as laser-induced fluorescence [8] or laser diode-based IR spectroscopy [9] have been proven in R\&D environments but remain too expensive for widespread production applications. Additionally, laser technology is a narrow band technique and this imposes limits on its broad application since full spectroscopic (broadband) sampling for CVD reactions is desirable for development work.

Conventional IR spectroscopy, on the other hand, is wellunderstood and relatively inexpensive with suitable detection limits, resolution and bandwidth and in principle it should be adaptable to use as a real-time monitor for CVD analyses. Additionally, modern Fourier Transform methods reduce the sampling time of the technique to a point where its use in control applications becomes feasible. Still, 
there is no commercial FT-IR appropriate in configuration and cost for use as an in situ sensor in these systems.

Specifically, the primary barrier to the use of FT-IR spectroscopy in this application is the lack of commercial optics to create an infrared probe beam that is suited for analysis of the boundary layer in CVD reactions. This layer, immediately above the wafer, typically has a thickness of $1-2 \mathrm{~mm}$; the chemistry in this region is the key to sensing the state of the deposition process. Solutions such as the use of long focal length mirrors to create a beam with small focal spot size and low beam divergence are unsuitable due to their impact on the (very limited) space around a reaction chamber. Using the insights gleaned from our modeling and experimental characterizations, we have developed IR optical designs that address these and other issues related to sampling production-style CVD reactors [12]. Our design (see Figure 2) reduces the infrared beam divergence and brings the size of the focal point to values comparable with that of the boundary layer. It includes configurations for the spectrometer that use a broadband source and an ellipsoidal cavitycoupling unit. This results in greater light throughput for the interferometer while maintaining nearly parallel beam input and output. The output beam from the interferometer is focussed and launched into a broadband mid-IR fiber bundle that carries the beam to the CVD chamber. After transiting the reaction chamber, additional optics collect and focus the beam on a detector. This novel optics design employs a conventional infrared light source to yield spectroscopically usable infrared intensities at the detector and smaller focal spot sizes. Our work to date has shown $2 \mathrm{~mm}$ diameter focal spots to be achievable with such a design [12]. This may be compared with a $10 \mathrm{~mm} \times 12 \mathrm{~mm}$ spot size for conventional FT-IR's, which is not useful for probing the boundary layer. This configuration has added potential for spatially resolved concentration analyses within the reactor. This aspect will be explored in future work where multiple beams (for gathering spatial distributions) and other innovations will be tested.

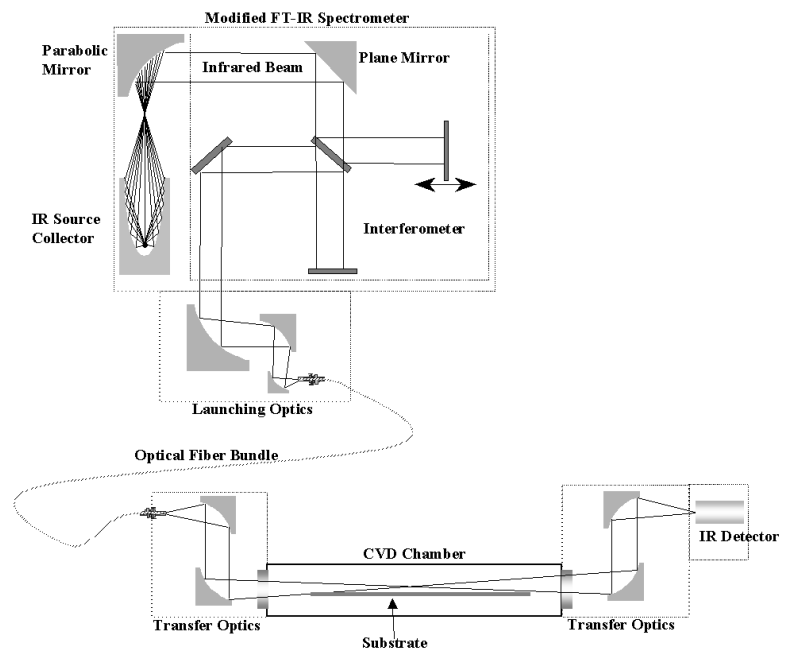

Figure 2: Modified FT-IR Optics for Sampling CVD Chambers

\section{CVD Process Control Technology}

A conventional PC-based process control system has been implemented on a home-built experimental CVD reactor in our laboratory. Together with the FT-IR sensor, this system achieves effective control of the chemistry of the process. The control hardware and software have been configured to accept real-time data from an FT-IR spectrometer that is interfaced with the reactor and analyzes the gas phase chemistry directly above the substrate surface. Tight real-time control of the reaction rate of tetraethoxysilane and ozone (TEOS/ $O_{3}$ ) using this in situ IR data has been demonstrated.

The development process for this system has clearly demonstrated the need for a higher level of automation, e.g., for intelligent supervisory control, and serves as a partial knowledge base for its implementation. The following discussion outlines the design and implementation of our conventional control system for CVD process control; subsequently, we provide a roadmap for future developments in Section 4

The first step toward the design of a control system for the boundary-layer chemistry, after implementation of a suitable sensor, was the determination of the process dynamics. Working from first principles is both time-consuming and unnecessary in this case, since, as in many manufacturing processes, the dynamics here are rather simple. They can be ascertained by traditional model identification approaches (see [22], or more simply [23]).

Typical results from one of the model identification exercises that we carried out are portrayed in Figure 3. The model obtained was determined using a basic least squares methodology [23]. The output of the identified system was the infrared absorption peak height in the vicinity of 1117 $\mathrm{cm}^{-1}$ that is recorded by the FT-IR sensor. This peak represents one of the absorption regions that is common to many CVD process gas analyses, and corresponds to overlapping bands from precursors and silicon-containing reaction intermediates that are important in characterizing the CVD process chemistry; its height was shown to have a linear correlation with the thin film deposition rate in earlier studies. The input to the process was a series of step changes in TEOS flow rate. As with many chemical/thermal processes, a first-order transfer function of the form $P_{k} / w_{T}=$ $K /(1+s T)\left(P_{k}=\right.$ peak height, reflecting the concentration of important gas-phase constituents, $w_{T}=$ TEOS flow rate; $K=$ process static gain and $T=$ a time constant) provides an adequate dynamic model. The input to the process was a "staircase" of TEOS flow, obtained by sending the corresponding set-point commands to the TEOS mass-flow controller; this staircase and resulting experimental data are depicted in Figure 3 . The identified model parameters are $\hat{K}=$ 0.32 and $\hat{T}=6 \mathrm{sec}$ for this experimental run. There may be a slight degree of nonlinearity, as suggested by the fact that the static gain seems to differ for the different step changes in TEOS flow rate; however, it is believed that much of the extra gain in taking the input step from 0.75 to 1.0 , for example, can be attributed to additional absorbance due to deposition on the chamber's windows after $300+$ seconds of reaction. There might also be a small ideal time delay (seen especially after the first step); however, the first delay may be due to the time required to initiate the deposition process. Based on these observations, the simple first-order transfer function was deemed an adequate representation for design of a basic conventional controller. 


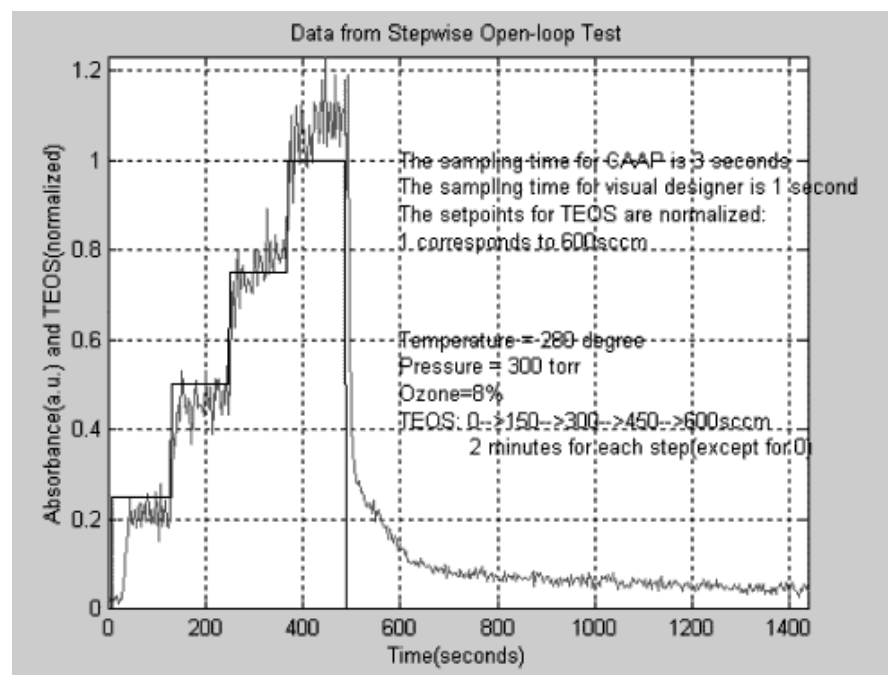

Figure 3: Input/Output Data and Model Identification Results

The prevalent control methods in process industries are proportional, proportional-integral and proportional-integralderivative algorithms (P, PI, PID) [24]. For this application PI control was used to achieve zero steady state error; addition of a derivative term (or rate feedback) might have been desirable if the process dynamics were more complicated, to improve transient response; however, this is not required for process dynamics as simple as those observed and identified for the CVD process at hand, so PID control was not considered.

The parameters of the PI controller were very simply set using a form of Ziegler-Nichols tuning [23]. Since the process model was somewhat rough and the performance requirements not too stringent, the proportional gain $K_{p}$ was set to 2 and the integral term time constant $T_{i}=5 \mathrm{sec}$. The design was validated by numerous of simulation studies, including investigations of sampling rate and noise levels over a number of expected operating conditions; sampling rate was of particular concern, given that the FT-IR sensor was slow and irregular in its sampling process.

The hardware platform for control system implementation was a standard PC and the software environment was Visual Designer [25]. An industry standard interface board was used to transfer the FT-IR sensor data to the control PC. Note that model identification was based on peak height, as mentioned previously; however, we switched to peak area for control, to achieve a better signal-to-noise ratio and greater robustness. Again, given the first-order process dynamics observed empirically, direct PI control was implemented in Visual Designer. The FT-IR data was made available at a rather slow and irregular 3-second sampling rate, and the control system ran at a slightly faster regular rate of one sample every two seconds. The process time constant is sufficiently slow (about $6 \mathrm{sec}$ ) that this proved adequate for good control.

The performance of this basic conventional control system is depicted in Figure 4. In this test/demonstration, the process was controlled for the first 60 seconds without FT-IR feedback, i.e., only the temperature, pressure and gas flow rates were controlled by their off-the-shelf controllers to follow their specified set points. Specifically, TEOS flow was set to $50 \%$ of it's maximum value, i.e., 0.5 on the normal- ized scale used in Figure 3. While these basic controllers are effective in their individual tasks (controlling temperature etc.) it is evident that the control of the CVD process chemistry is quite loose, as indicated by the high degree of "wander" in the FT-IR peak-area signal. At $\mathrm{t}=60$ seconds the FT-IR control loop is closed, and the process settled to the specified peak-area setpoint 0.8 with a time constant of about 20 seconds. As a further test of closed-loop control of the CVD process chemistry, the peak-area setpoint was changed from 0.8 to 0.5 at $\mathrm{t}=245 \mathrm{sec}$. The process responded with a small delay (about $5 \mathrm{sec}$, probably due in large part to the long FT-IR data cycle time) and a time constant of about $20 \mathrm{sec}$, which is appropriate for a process which is quite slow, as indicated by the identified model, and a conservatively tuned controller.

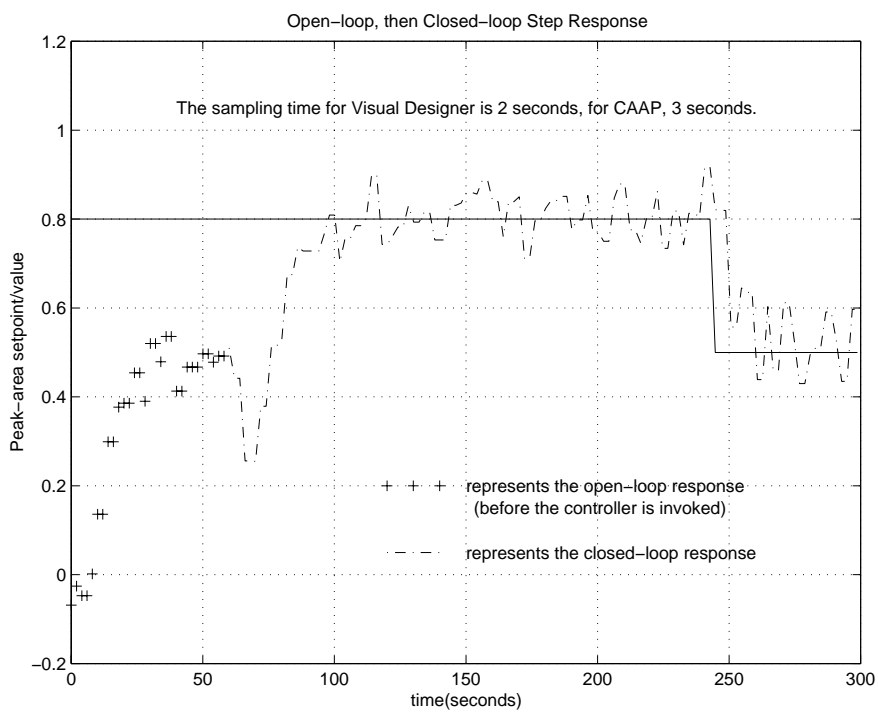

Figure 4: Open- and Closed-Loop CVD Process Variations

Visual Designer was used as the software platform for building this control system, for its direct support for the implementation of a conventional control algorithm and for its convenient graphical editor for creating a graphical user interface (GUI) for the operator. For the development system this GUI was devised to be simple to operate and intuitive in its use. A sample screen display during operation is portrayed in Figure 5.

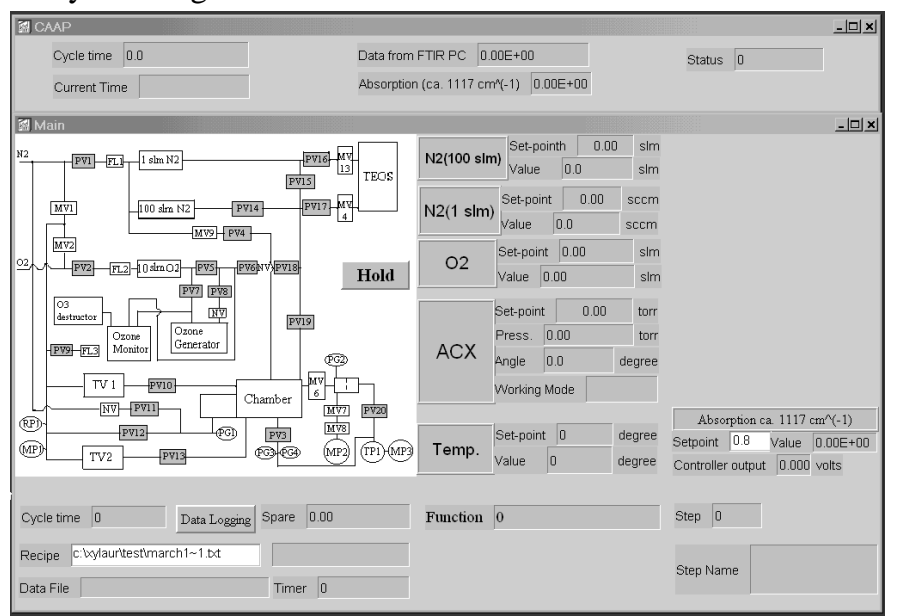

Figure 5: Graphical User Interface for Real-Time Process Control 


\section{Advanced CVD Process Control}

Standard control practice for CVD tools uses local loops to regulate the basic process variables, i.e., pressure, temperature, gas flow rates, etc. to follow predetermined recipe profiles or schedules. The simple addition of the conventional control system described above using in situ process chemistry monitoring and closing the loop produces significantly better control of the CVD process chemistry. This and still greater degrees of process control will be needed for processing larger substrates with ever smaller feature sizes and tighter materials specifications. However, this conventional approach involves intensive and time-consuming manual operations that are unsuitable for commercial applications, including:

1. determining the relations between basic process manipulations (gas flows, pressure, temperature) and the process chemistry and thin-film properties,

2. determining the appropriate control architecture, i.e., which sensed variables to use for the control of which process or film property, and

3. designing, parametrizing and implementing the final control algorithms, including testing and tuning the controller.

The work involved in step 1 is typically empirical. Development of the conventional (fixed) supervisory control system operations, steps 2 and 3, involves a substantial amount of manual data collection and decision making, e.g., investigating process correlations, identifying process dynamic models and deciding on the control system architecture and design. This state of affairs is appropriate for research and development, but is not conducive to the introduction of effective and flexible commercial controllers for advanced semiconductor manufacturing. A substantial amount of this burden (but by no means all) can and should be assumed by a more capable intelligent supervisory control strategy. Such strategies have been conceived and developed in other applications [13] but are new to the semiconductor manufacturing industry.

In the next stage of our research we will develop an intelligent supervisory control system that can automate, to a considerable degree, the manual process optimizations and tunings needed for control in emerging semiconductor technologies. The system will expand the operating envelope for well-understood processes such as TEOS/ $\mathrm{O}_{3} \mathrm{CVD}$ and facilitate implementation for new ones. The control system will incorporate automatic model identification methods (using on-line data and recursive algorithms [22]) to accommodate, for example, new temperature and pressure regimes and to learn to handle a new process, given an appropriate starting point and high-level direction from the process scientist. In this way, built-in models of the process will be continually refined and the control algorithm tuned accordingly. These models will also serve as the basis for process optimization and control system tuning. Such a system is designated as an intelligent self-optimizing control (ISOC) system.

A schematic of the ISOC architecture is shown in Figure 6. It will automate system configuration and incorporate learning mechanisms that adapt to new conditions and requirements, and generate optimized model-based recipes. The learning mechanisms involve the feedback of process chemistry (concentrations) and quality (uniformity, conformability, etc.) variables to the model refinement module to tune and update the models. This ISOC architecture is based on a concept originally developed for metal-matrix composite fabrication [13]. The specific functions and details of the system will be developed for this new application.

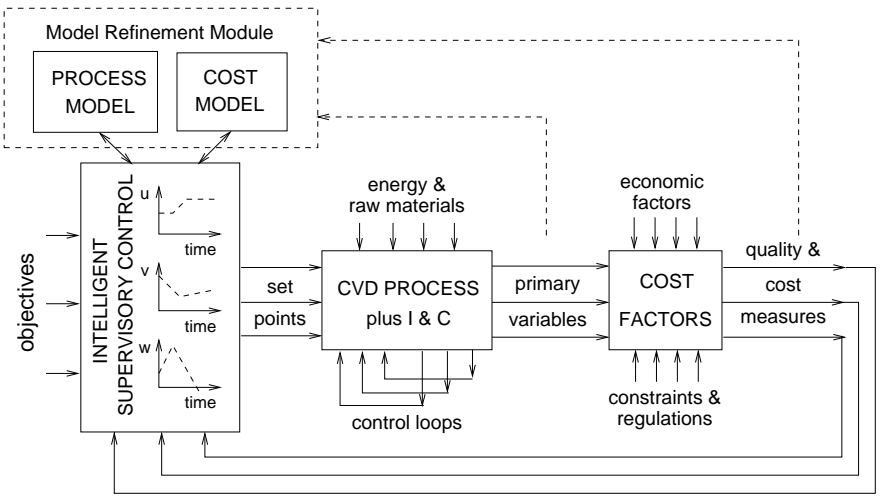

Figure 6: Architecture for intelligent Self-Optimizing Control

This ISOC system development will be undertaken by:

- Step 1: capturing the knowledge gained in the current project and automating it as appropriate

- Step 2: implementing automatic control system design and/or tuning methods for the feedback loops,

- Step 3: developing a model-based recipe optimization approach, to generate set-point profiles (time sequences to be executed by the control system, and

- Step 4: introducing learning algorithms to improve the process models as the system operates.

The technical approach for the first step above involves combining the experience gained in previous work with information from the experimental characterization research and the fundamental modeling effort (i.e., structure and approximate parameter values for a simplified process model suitable for execution in real time), design of experiments methods [14], automatic model identification techniques [15] and simple expert system methodologies to develop automatic procedure for process observation and manipulation of the results via the in situ FT-IR sensor. Given step-wise changes of the process manipulations the system will infer steady-state correlations and approximate dynamic behavior (e.g., time delay and lag); this is adequate for the automatic synthesis of control architectures and algorithms.

The basic process model information gained from Step 1 will be used as the basis for automatic control system design and/or tuning. The approach will be based on proven methods for automatic control system synthesis, including robust frequency-domain approaches [16], linear controller auto-tuning approaches [17], and, if required, extensions for nonlinear controller autosynthesis [18]. The incorporation of a model-based optimization module to generate recipes for the process (Step 3) will involve a second small expert system to set up and execute straightforward optimization problems. The heuristics in the expert system will be based on the experience gained in implementing earlier systems. These problems will be solved by standard methods 
$[19,20]$, the result being a parametric definition of setpoint profiles to be regulated by conventional control loops.

The approach to Step 4 will be to build an expert system combining classical on-line model identification techniques (time-series analysis of input and output data to infer loworder linear dynamic models [15]) with perhaps neural net methods [21] to continually refine the known nonlinear dynamic relations between variables. Both of these methodologies are sufficiently well understood and amenable to automation that the development of such an expert system is feasible. The result will be a self-tuning nonlinear multiinput/multi-output (MIMO) model for the process, describing how the process manipulations effect the process chemistry and the physical properties of the final product. The operation can be completely automatic (in real time) for the CVD PROCESS block in Figure 6. The automation of this process for the COST FACTORS block will probably require the characterization of the properties of the end product to be done off-line and fed back to the system. This learning capability will allow the system to accommodate initial uncertainty (e.g., imprecise knowledge of the process dynamic interactions) and system changes over time (e.g., hardware modifications that are not too drastic, changes due to wear, fouling etc.), thereby improving its performance (e.g., the quality of the product).

\section{Summary and Conclusions}

Over the past three years we have built and equipped a CVD research laboratory in our facility. The laboratory has capabilities for the modeling of CVD reaction chemistries and the experimental confirmation of these models, the optimization of in situ spectroscopic probes of CVD reactions, and the development of systems that use in situ analytical data for real-time process control. We have carried out spectroscopic studies in a production-style CVD chamber that have demonstrated the feasibility of our approach as an in situ probe of CVD reactions in general and of the tetraethoxysilane/ozone $\left(\mathrm{TEOS} / \mathrm{O}_{3}\right)$ process in particular $[10,11,12]$.

Our results confirm the sensitivity of an FT-IR sensor for quantitative analyses, and we have demonstrated correlations between the gas-phase infrared signatures and the physical and chemical properties of the thin film produced in the reaction. We have developed chemical kinetic models of CVD reactions [12] which, while consistent with previous work $[5,6]$, improve upon the existing models of TEOS $/ \mathrm{O}_{3}$ CVD. These models have provided us with effective guidance in the design of novel optics configurations for probing the reaction boundary layers in the CVD reactions that have been modeled. Using the insights gained from our modeling and experimental characterizations, we have developed IR optical designs that address these and other issues in the practical sampling of production-style CVD reactors [12].

Finally, we have successfully developed a simple conventional control system to close the loop around the process chemistry, for one challenging process. Based on this accomplishment, we have devised a plan for the next generation more flexible and functional control approach for commercial applications.

\section{References}

[1] The National Technology Roadmap for Semiconductors, published by the Semiconductor Industry Association, 1997.

[2] T. R. Hogness, T. L. Wilson and W. C. Johnson, J. Am. Chem. Soc., 58, 108 (1936).

[3] H. K. Moffat, K. F. Jensen and R. W. Carr, J. Phys. Chem., 95, 145 (1991).

[4] W. L. M. Weerts, M. H. J. M. de Croon and G. B. Marin, J. Electrochem. Soc., 144, 3213 (1997).

[5] D. M. Dobkin, S. Mokhtari, M. Schmidt, A. Pant, L. Robinson and A. Sherman, J. Electrochem. Soc., 142, 2332 (1995).

[6] W. N. Gill and S. Ganguli, J. Vac. Sci. Technol., B15, 948 (1997).

[7] I. Nir, J. Winniczek, M. P. Splichal, H. M. Anderson and A. Stanton, Photonics Spectra, 27, 7 (1993).

[8] G. A. Hebner, J. Appl. Phys., 80, 2624 (1996).

[9] J. E. Butler, N. Bottka, R. S. Sillmon and D. K. Gaskill, J. Crystal Growth, 77, 163 (1986).

[10] T. K. Whidden and S. Doiron, Characterization and Metrology for ULSI Technology, 1998 International Conference, edited by D. G. Seiler, A. C. Diebold, W. M. Bullis, T. J. Shaffner, R. McDonald and E. J. Walters, the American Institute of Physics, p. 459 (1998).

[11] T. K. Whidden, S. Y. Lee and S. Doiron, Dielectric Material Integration for Microelectronics, The Electrochem. Soc., Pennington, NJ, PV98-3, 326 (1998).

[12] T. K. Whidden, S. Y. Lee, X. Bao, M. Couturier, J. H. Taylor, P. Lu, S. Romet and Z. Xiaozhong, Fundamental Gas-Phase and Surface Chemistry of Vapor Phase Materials Synthesis, The Electrochem. Soc., Pennington, NJ, in press.

[13] J. H. Taylor, L. P. Harris, P. K. Houpt, H. P. Wang and E. S. Russell, Advanced Sensing, Modeling and Control of Materials Processing, edited by E. F. Matthys and B. Kushner, TMS Publications, Warrendale, PA, p. 275 (1991).

[14] N. Belaverdram, Quality by Design: A System for Experimentation for Industrial Research, Prentice Hall, 1995.

[15] W. E. Larimore, preprint from The 11th IFAC Symposium on System Identification, July 8-11, Fukuoka, Japan, Vol. 1101-6 (1994).

[16] S. Engell and R. Mueller, Proc. ESCAPE-1, Computational Chemical Engineering, 16, 157 (1992).

[17] K. J. Åström and T. Hägglund, Proc. IFAC 9th World Congress, Budapest, Hungary, 1984.

[18] J. H. Taylor and P. G. Stringer, Int. J. Adaptive Control and Signal Processing, 5, 21 (1991).

[19] D. P. Bertsekas, Constrained Optimization and Lagrange Multiplier Methods, Academic Press, New York, 1982.

[20] N. Ansari and E. Hou, Computational Intelligence for Optimization, Kluwer Academic Publishers, 1997.

[21] M. Brown and C. Harris, Neurofuzzy Adaptive Modelling and Control, Prentice Hall, New York, 1994.

[22] L. Ljung, System Identification - Theory for the User,Prentice-Hall, Englewood Cliffs, NJ., 1987.

[23] K. J. Astrom and B. Wittenmark, Computer Controlled Systems Theory and Design, Prentice-Hall, Englewood Cliffs, NJ., 1984.

[24] C. D. Johnson, Process Control Instrumentation Technology, Prentice Hall, Upper Saddle River NJ, 1997.

[25] Intelligent Instrumentation (a Burr-Brown Company), Getting Started with Visual Designer, 1998. 